\title{
Usage élargi du modafinil : quel risque addictif ?
}

\section{Broadening prescription of modafinil : is the risk of addiction increased?}

\section{Hélène EYSSERIC ${ }^{*(1,2)}$, Françoise VINCENT ${ }^{(1)}$, Michel MALLARET $^{(3)}$, Claude-Elisabeth BARJHOUX ${ }^{(3)}$, Luc BARRET ${ }^{(2)}$}

(1) UF de Pharmacologie-Toxicologie, Département de Biologie et Pathologie de la Cellule, CHU de Grenoble

BP 217 - 38043 Grenoble Cedex 9

(2) Laboratoire de Médecine Légale, UFR de Médecine, Domaine de la Merci - 38706 La Tronche Cedex

(3) UF de Pharmacovigilance-Pharmacodépendance, Département de Biologie et Pathologie de la Cellule, CHU de Grenoble - BP 217 - 38043 Grenoble Cedex 9

*Auteur à qui adresser la correspondance : Hélène EYSSERIC, Laboratoire Pharmacologie-Toxicologie, Département de Biologie et Pathologie de la Cellule, CHU de Grenoble - BP 217 - 38043 Grenoble Cedex 9 Tél : 0476765492 - Fax : 0476768938 -E-mail : HEysseric@chu-grenoble.fr

(Reçu le 20 janvier 2006 ; accepté le 14 février 2006)

\section{RÉSUMÉ}

Le modafinil est un psychostimulant commercialisé en France depuis 1994 sous le nom de Modiodal@. Son mécanisme d'action, différent de celui des amphétamines, repose sur une potentialisation de l'activité $\alpha-1$-adrénergique cérébrale et sur l'inhibition de la recapture de la dopamine. Chez l'hommé, le modafinil est un médicament éveillant qui modifie les paramètres électrophysiologiques reflétant la vigilance et s'oppose à la diminution des performances cognitives (notamment mnésiques), psychomotrices et neurosensorielles induites par la privation de sommeil. Ses principales indications comprennent le traitement de la narcolepsie ou de l'hypersomie idiopathique et depuis peu l'hypersomnolence liée à l'apnée du sommeil.

Compte-tenu des propriétés de ce produit, on peut donc craindre son détournement d'usage et un abus de consommation. Son pouvoir toxicomanogène semble faible à doses thérapeutiques (200 à $400 \mathrm{mg} / \mathrm{j}$ ) mais fait encore l'objet d'évaluations à doses plus élevées. C'est pourquoi le

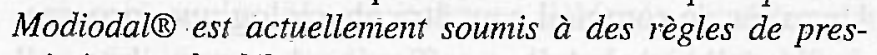
cription et de déliviance particulières très strictes : pres-

\section{SUMMARY}

Modafinil is a psychostimulant commercialised in France under the name of Modiodal $($, since 1994. Its mechanism of action, different from that of amphetamines, rests on a potentiation of cerebral $\alpha$-1-adrenergic activity and inhibition of dopamine recapture. Modafinil is a drug which action results in "waking you up" by modifying electro physiological parameters controlling vigilance. It acts against the reduction of neuro-sensorial, cognitive (in particular muemonic) and psychomotor performance, induced by deprivation of sleep. Its major indications concern narcolepsy, idiopathic hypersomnia and, recently, hypersomnolence related to sleep apnea.

Therefore knowing the properties of this product, one can worry concerning its possible diversion of use, addiction and abuse. Its toxicomanogenous potential seems to be rather limited at therapeutic dosage (200 to $400 \mathrm{mg} /$ day) but is still subject to evaluation for higher dosage. That's why very strict rules of prescription and delivery are maintained in France : initial prescription is reserved to neurologists, pneumologists and practitioners of sleep centers. 
cription initiale annuelle réservée aux spécialistes en neurologie ou en pneumologie et aux médecins exerçant dans les centres de sommeil.

L'analyse dans les milieux biologiques du modafinil et de ses métabolites (décrits comne inactifs : modafinil acide et modafinil sulfone) est majoritairement proposée par chromatographie liquide couplée à différents modes de détection (UV ou spectrométrie de masse). Sa thermolabilité rend difficile son analyse par chromatographie gazeuse. Les concentrations plasmatiques d'ordre thérapeutique en modafinil sont décrites entre 4 et $13 \mathrm{mg} / \mathrm{L}$ environ.

Les cas cliniques d'intoxication au modafinil sont rarissimes. En revanche, des cas de dopage ont été rapportés dans le milieu de l'athlétisme en 2003. De nombreux essais cliniques sont en cours pour évaluer de nouvelles indications telles que le trouble déficitaire de l'attention chez l'enfant ou encore la fatigue associée à des pathologies telles que la sclérose en plaque ou la dépression.

Face à un risque de conduite addictive, nul doute que la vigilance concernant le modafinil devra restée constante, si son usage devait être élargi.

MOTS-CLÉS

Modafinil, addiction, narcolepsie, psycho stimulant.

\section{Introduction}

Dans la course à la performance, la tentation peut être grande de vouloir augmenter sa vigilance et ses facultés intellectuelles ou au contraire de vouloir diminuer son temps de sommeil (1). La consommation de psychostimulants peut devenir abusive et parmi ceux-ci un médicament comme le Modiodal® (modafinil) (2), indiqué pour le traitement de deux maladies neurologiques, la narcolepsie et l'hypersomnie idiopathique, et plus récemment, indiqué pour le traitement en seconde intention de la somnolence diurne excessive résiduelle associée au SAHOS (syndrome d'apnées/hypopnées obstructives du sommeil), doit faire l'objet d'une attention particulière vis-à-vis du risque d'abus ou de détournement d'usage.

Après 12 ans de commercialisation de cette molécule au mode d'action tout à fait original et découverte par un laboratoire français (laboratoire Lafon), il nous a paru intéressant de rappeler les principales caractéristiques pharmacologiques et toxicologiques de ce produit psycho-actif, de faire le point des connaissances actuelles sur son potentiel de pharmacodépendance et de tolérance et d'attirer l'attention des toxicologues analystes sur son dépistage. Les méthodes de dosage les plus récentes sont également présentées, cependant très peu de cas cliniques d'intoxication sont répertoriés. Hormis son usage thérapeutique, volontairement très « cadré » par les Autorités Sanitaires Françaises (produit soumis à la législation des médicaments d'exception à prescription restreinte), le modafinil a été impli-
The main technique of dosage of modafinil and its metabolites (modafinil acid and modafinil sulfonic which are, by the way, described as inactive) in biological fluids, is liquid chromatography (coupled with UV or mass spectrometry). The thermolability of these molecules makes the use of gas chromatography difficult. For Modafinil, therapeutic plasma concentrations range from 4 to $13 \mathrm{mg} / \mathrm{L}$, approximately.

Clinical cases of modafinil intoxication appear to be extremely rare.

On the other hand, cases of doping in athletics athletes were brought back in 2003.

Many clinical trials are currently conducted to evaluate new indications such as child attention disorder or tiredness associated with pathologies such as multiple sclerosis or depression.

In front of a clear risk of addictive behaviour, there is no doubt that a high degree of vigilance will have to be maintained if the usage of modafinil was to be widened.

\section{KEY-WORDS}

Modafinil, addiction, narcolepsy, psychostimulant.

qué dans des cas de dopage sportif dans le domaine de l'athlétisme. Il est testé dans de nombreuses autres indications tant chez les militaires pour améliorer leur résistance que dans d'autres affections neurologiques. Une augmentation de sa consommation devra nous inciter à encore plus de vigilance vis-à-vis du risque potentiel de conduite addictive.

\section{Mécanismes de l'éveil (3)}

L'éveil correspond au passage de l'état de sommeil à l'état de veille.

Il est orchestré par des noyaux gris centraux et par la formation réticulée du tronc cérébral. Il se traduit par l'apparition d'ondes désynchronisées courtes et rapides à l'électro-encéphalogramme (EEG).

Il n'y a pas un mais des systèmes d'éveil mettant en jeu de nombreux transmetteurs (dopamine, acétylcholine, noradrénaline, adrénaline, histamine et des acides aminés tels que le glutamate et l'aspartate).

On distingue l'éveil cortical : apparition d'ondes désynchronisées courtes et rapides à l'EEG, à l'origine du comportement d'éveil, et l'éveil comportemental : augmentation du tonus musculaire, de la fréquence cardiaque et respiratoire, et de la pression artérielle.

\section{Les hypersomnies}

Un excès de sommeil peut être physiologique (grossesse, liés à l'âge), induit (insuffisance de sommeil, travail 
posté, vols transméridiens, alcool, médicaments), lié à une pathologie organique reconnaissable (apnée du sommeil, maladies psychiatriques, neurologiques, infectieuses...) ou primaire (narcolepsie, hypersomnie idiopathique).

\section{La narcolepsie (4) :}

C'est une maladie encore peu connue mais non exceptionnelle (prévalence de 25 à 35 pour 100000 selon les enquêtes). Elle débute de l'enfance jusqu'à l'âge de 50 ans avec un pic dans la deuxième décennie. Elle est caractérisée par deux signes cardinaux, les accès de sommeil irrésistibles (quotidiens ou pluri-quotidiens, à horaire fixe pour un même patient, restaurateurs d'une vigilance normale) et les attaques de cataplexie ou perte soudaine de tonus musculaire à déclenchement émotionnel (elles peuvent être partielles et ainsi ne toucher que certains muscles, ou totales, entrânant une chute).

Les autres signes dits accessoires parce qu'inconstants sont les hallucinations hypnagogiques (à l'endormissement), les paralysies du sommeil et le mauvais sommeil.

Les hallucinations (essentiellement auditives parfois visuelles) sont désagréables, à tel point anxiogènes que le patient appréhende de se coucher (sensation d'une présence menaçante dans la chambre, impression de s'endormir sur une carapace de crocodile...).

Son diagnostic repose sur l'identification des signes cardinaux. Des examens complémentaires sont nécessaires pour le confirmer avant la mise en œuvre d'un traitement destiné à durer toute la vie. Ils comprennent un enregistrement poly-somnographique de nuit et de jour montrant les endormissements directs en sommeil paradoxal, un génotypage montrant le plus souvent une association des antigènes d'histocompatibilité HLA DQB $1 * 0602$ et dans les cas douteux, un dosage d'hypocrétine-1 du liquide céphalorachidien (LCR) révélant des concentrations inférieures à $110 \mathrm{pg} / \mathrm{mL}$ hautement spécifiques de narcolepsie avec cataplexie.

Dans la moitié des cas, il est retrouvé un facteur déclenchant tels qu'un stress psychologique, un traumatisme crânien, une grossesse, une maladie infectieuse,,$\ldots$

La connaissance de la physiopathologie doit beaucoup à l'existence d'un modèle animal naturel, le chien narcoleptique. Les accès de sommeil et les cataplexies ont un contrôle pharmacologique différent, les systèmes dopaminergiques agissent sur les premiers et les systèmes noradrénergiques sur les seconds. Outre les données concernant l'association HLA DQB $1 * 0602$, un mécanisme auto-immunitaire est à l'étude actuellement avec la découverte d'une mutation du récepteur- 2 de l'hypocrétine chez le chien narcoleptique et l'absence d'hypocrétine-1 dans le LCR de $90 \%$ des malades (4). $\mathrm{La}$ narcolepsie est donc une maladie multifactorielle avec une composante environnementale et une composante génétique qui a un lourd impact socio-professionnel pour le patient. Sur le plan professionnel, les accès de somnolence diurne aboutissent à des licenciements, des difficultés d'embauche et expose le patient à un risque accru d'accident du travail et de la circulation. Au niveau social, la narcolepsie peut conduire à un isolement et retentir de façon négative sur la cellule familiale. Une pathologie psychiatrique à type de dépression peut être engendrée.

Chez l'enfant, la narcolepsie est responsable de retards scolaires irréversibles.

\section{L'hypersomnie idiopathique}

Elle est plus rare que la narcolepsie et correspond à une hypersomnie isolée sans cause connue. Elle correspond à une somnolence diurne excessive quasi-permanente (à l'inverse des narcoleptiques qui sont soit vigilants soit somnolents). Sur ce fond, apparaissent des accès de sommeil moins irrésistibles que dans la narcolepsie mais de longue durée et non restaurateurs d'une vigilance normale. Les patients hypersomniaques ne présentent pas d'endormissement en sommeil paradoxal.

\section{Somnolence diurne excessive résiduelle associée au syndrome d'apnées/hypo- pnées obstructives du sommeil (SAHOS)}

Cette pathologie constitue une nouvelle indication rajoutée en juin 2004 lors de la révision de l'AMM du Modiodal( (2). Il est précisé que ce traitement ne pourra s'appliquer que si la somnolence persiste malgré l'application techniquement correcte pendant le sommeil d'un traitement mécanique bien suivi (Pression Positive Continue par voie nasale ou nasobuccale) corrigeant les évènements respiratoires éveillants. Par ailleurs une confirmation du diagnostic doit être établi par un enregistrement polygraphique au cours du sommeil.

\section{Le mécanisme d'action du modafinil}

D'après la mise au point récente de Billiard dans la Revue de Neurologie (5), le mécanisme d'action du modafinil n'est pas encore connu avec précision. Bien que les effets du modafinil soient réduits par la prazosine, un antagoniste des récepteurs $\alpha-1$ adrénergiques, et que le produit soit encore catalogué comme potentialisateur indirect de l'activité des synapses $\alpha-1$ adrénergiques, le modafinil n'est pas un agoniste $\alpha-1$ adr- 
énergique direct ou indirect. Deux études récentes ont souligné le rôle de la dopamine. Une première étude faite chez le chien narcoleptique a comparé l'activité d'inhibiteurs de la recapture de la dopamine, de la noradrénaline, de la d-amphétamine et du modafinil sur la veille, et a montré que la puissance in vivo des inhibiteurs de la recapture de la dopamine et du modafinil sur la veille était significativement corrélée à leur affinité in vivo pour le transporteur de la dopamine (DAT) tandis que les inhibiteurs de la recapture de la noradrénaline avaient peu d'effet sur la veille. Une deuxième étude a montré que des souris knock-out pour le gène du transporteur de la dopamine ne répondaient pas à l'action éveillante du modafinil, de la metamphétamine et d'un bloqueur sélectif du transporteur de la dopamine le GBR 12909, indiquant par là le rôle clé joué par le transporteur de la dopamine dans l'action éveillante des amphétamines et du modafinil. De plus, l'action du modafinil est associée à une diminution de la libération du GABA dans les régions du système nerveux central aptes à jouer un rôle dans le sommeil et la veille. Dans une étude mesurant l'expression de $c$-fos (mesure l'activité neuronale) chez le chat, les amphétamines et le méthylphénidate produisent une activation diffuse du striatum, du cortex et du noyau accubems, tandis que le modafinil entraîne une activation relativement spécifique de sites de l'hypothalamus antérieur qui pourraient être impliqués dans les mécanismes du sommeil et de l'éveil. Enfin une activation des neurones à orexine de l'hypothalamus latéral a été mise en évidence chez des souris traitées par modafinil et sacrifiées deux heures plus tard.

\section{Principales caractéristiques physico-chimiques modafinil $(6,7)$}

Dénomination chimique : DL-2-[(diphénylméthyl)sulphinyl] acétamide

ou 2-(benzydrylsulfinyl) acétamide

Forme moléculaire : $\mathrm{C}_{15} \mathrm{H}_{15} \mathrm{NO}_{2} \mathrm{~S}$

Poids moléculaire : 273,36

Le modafinil est une poudre blanche cristalline, soluble dans le méthanol, peu soluble dans l'éthanol et insoluble dans l'eau.

Son point de fusion se situe entre 150 et $155^{\circ} \mathrm{C}$ avec décomposition thermique.

Sa formule présente un atome de soufre asymétrique entrainant une stéréo-isomérie. Le modafinil correspond en fait au mélange racémique, les deux énantiomères $\mathrm{D}$ et $\mathrm{L}$ étant biologiquement actifs.

\section{Eléments de pharmacoci- nétique $(2,5,6,8)$}

La résorption orale du modafinil est lente et entraîne un pic sanguin (Cmax) compris entre 2 et 4 heures. La prise de nourriture ne modifie pas la biodisponibilité du modafinil, en dehors d'un léger décalage de la Cmax d'environ 1 heure. Son volume de distribution est relativement faible d'environ $0,9 \mathrm{~L} / \mathrm{Kg}$. Le pourcentage de liaison aux protéines plasmatiques (principalement l'albumine) est d'environ $60 \%$. Il subit une métabolisation hépatique importante environ de $90 \%$ (voir figure 1) par déamination (estérase), oxydation (CYP3A4/5) et hydroxylation conduisant à des métabolites inactifs (modafinil acide majoritaire, et modafinil sulfone) éliminés par voie urinaire. Seule $10 \%$ d'une dose de modafinil est excrétée inchangée dans les urines. La demi-vie d'élimination après une dose orale unique de 200 ou $400 \mathrm{mg}$ est de 10 à 13 heures. L'état d'équilibre plasmatique est atteint après 3 jours de prise quotidienne de 200 à $400 \mathrm{mg}$ de modafinil.

La demi-vie d'élimination de l'énantiomère $\mathrm{D}$ est plus courte que celle de l'énantiomère $L$ ce qui permet de supposer un mécanisme d'élimination stéréospécifique.

Des études in vitro sur hépatocytes humains ont montré que le modafinil induit faiblement, et de façon dépendante de la concentration, les isoenzymes CYP1A2, CYP2B6 et CYP3A4. L'activité du CYP2C9 hépatocytaire est inhibée.

Sur des microsomes hépatiques humains, le modafinil et le modafinil sulfone provoquent une inhibition partielle, compétitive et réversible du CYP2C19 à des concentrations correspondant aux doses thérapeutiques (200-300 mg/j).

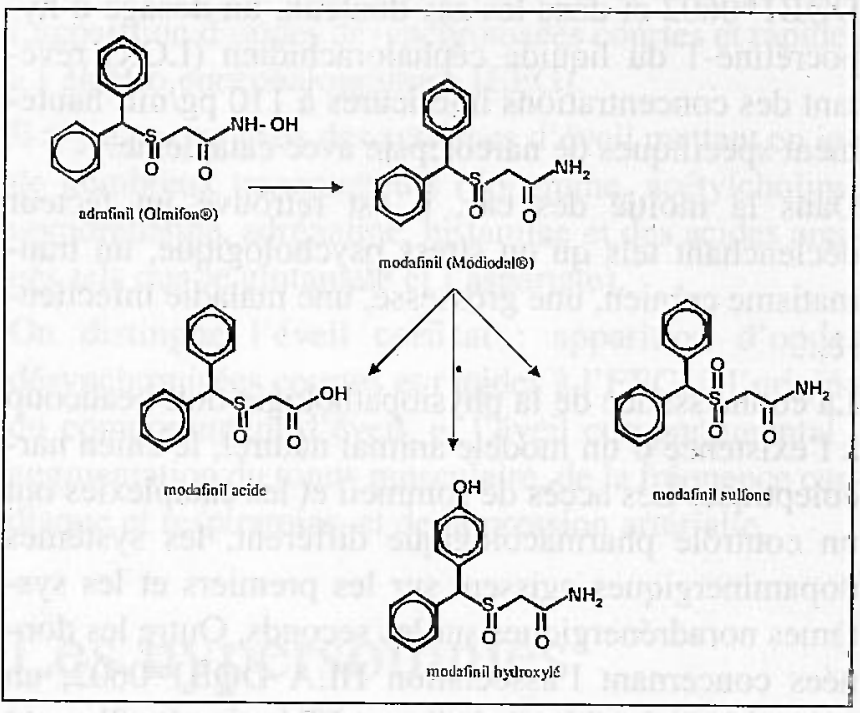

Figure I : Biotransformation du modafinil d'après le laboratoire Lafon (13). 


\section{Effets cliniques du modafi- nil $(2,3,5)$}

Chez l'homme, le modafinil est un médicament éveillant qui en fonction de la dose administrée, modifie les paramètres électrophysiologiques reflétant la vigilance et s'oppose à la diminution des performances cognitives (notamment mnésiques), psychomotrices et neurosensorielles induites par la privation de sommeil.

Son effet sur les performances cognitives pourrait être variable, notamment selon le quotient intellectuel (9).

Administré le soir, le modafinil retarde l'endormissement et diminue la durée totale de sommeil.

Les effets psychostimulants du modafinil sont plus intenses que ceux de-la caféine mais moindres que ceux de l'amphétamine (10).

Par rapport aux substances amphétaminiques qui sont les seuls médicaments capables d'induire des effets éveillants du même ordre, le modafinil exerce peu ou pas d'effet sur le système nerveux autonome, en particulier sur les chiffres de pression artérielle dont l'augmentation est nulle ou cliniquement peu pertinente.

L'insuffisance hépatique entraîne une augmentation de la Cinax et de la demi-vie d'élimination pas diminution de sa biotransformation en dérivé acide.

\section{Effets indésirables (11)}

La plupart des effets indésirables semblent liés à un effet pharmacodynamique éveillant ou stimulant trop important. On observe alors une insomnie, une anorexie, une sensation de tension interne, une excitation, voire de l'agressivité. Ces symptômes peuvent disparaître avec le temps mais peuvent nécessiter une diminution de posologie.

\section{Contre-indications $(2,11)$}

Elles comprennent les cas d'anxiété généralisée, la grossesse en l'absence de données suffisantes pour un éventuel effet malformatif et la pratique des sports de compétition.

\section{Interactions médicamen- teuses (2)}

Avec la ciclosporine : risque en raison de son potentiel inducteur enzymatique de diminution des concentrations sanguines et de l'efficacité de l'immunosuppresseur.

Avec les contraceptifs oraux : risque de diminution de l'effet contraceptif.

\section{Surdosage (2)}

Les symptômes accompagnant le plus souvent un surdosage en modafinil, seul ou associé à d'autres médicaments ont été les suivants : symptômes neurologiques centraux tels qu'insomnie, agitation, désorientation, confusion, excitation et hallucinations ; symptômes digestifs tels que nausées et diarrhées ; et signes cardiovasculaires tels que tachycardie, bradycardie, hypertension artérielle et douleur thoracique.

Conduite à tenir : hospitalisation, lavage gastrique et surveillance de l'état psychomoteur ; monitorage ou surveillance cardiovasculaire pendant 48 heures.

\section{Potentiel de pharmacodé- pendance}

Sa structure chimique de type benzhydrylsulfinyl-acétamide lui confère une faible hydrosolubilité et une instabilité à haute température qui limitent ainsi son mésusage par injection ou par inhalation (12).

Chez l'animal, une étude d'auto-administration expérimentale chez le singe révèle un potentiel de pharmacodépendance équivalent à celui de la cocaïne (13). En revanche, les études chez l'animal (singe et souris) n'ont pas permis de mettre en évidence un syndrome de sevrage $(13,14)$.

Chez l'homme, à doses thérapeutiques, le potentiel de pharmacodépendance du modafinil semble faible comparé à celui de l'amphétamine. En particulier, le modafinil n'exerce pas de comportement appétitif (modification comportementale aiguë entraînant un désir de reprendre la substance), contrairement à l'amphétamine. Cependant le modafinil est éveillant chez tout le monde et pas seulement chez les narcoleptiques (11). On peut donc craindre une utilisation du modafinil par des sujets sains désirant augmenter leurs performances en réduisant leur temps de sommeil ou maintenir un haut niveau d'éveil lors de tâches longues et monotones. On peut rappeler que réduire son temps de sommeil de façon prolongée n'est pas anodin pour un individu (diminution des ses défenses immunitaires) et que par ailleurs les médicaments éveillants peuvent induire un état de tension interne.

A doses plus élevées, le potentiel de pharmacodépendance du modafinil fait encore l'objet d'évaluations. Une étude du modafinil à une posologie jusqu'à $800 \mathrm{mg}$ a été réalisée par comparaison au méthylphénidate comme référence et démontre un potentiel de pharmacodépendance moindre (15).

Le principal risque du modafinil réside en un risque de mésusage s'il est prescrit abusivement dans des situations autres que la narcolepsie. 


\section{Règles de prescription en France $(2,11)$}

Compte-tenu des propriétés de ce produit, on peut donc craindre, d'un point de vue santé publique, un détournement d'usage et un abus de consommation. C'est pourquoi les Autorités Sanitaires Françaises ont souhaité, dès le début de la commercialisation, surveiller de près la prescription de ce psychostimulant. Le Modiodal@ est ainsi un médicament soumis à prescription restreinte dont les conditions de prise en charge relèvent des médicaments d'exception. L'autorisation de mise sur le marché classe le Modiodal ${ }^{\circledR}$ dans la catégorie des médicaments à prescription initiale hospitalière annuelle réservée aux spécialistes et/ou services spécialisés en neurologie et/ou aux centres du sommeil.

En outre, pour des raisons médico-économiques (coût du traitement journalier au Modiodal@ indiqué dans le Vidal $2005: 6,37$ à 12,74 euros), le remboursement aux assurés sociaux est subordonné à la procédure relative aux médicaments d'exception (art. R. 163-2 du code de la sécurité sociale).

D'après le bilan annuel 2003 des vigilances de l'AFSSAPS (16), le Modiodal ${ }^{\circledR}$ reste sous surveillance particulière car il conserve un important potentiel d'abus et il a été constaté que le remboursement n'avait pas été demandé pour une part importante de ses ventes (de l'ordre de 20 à $40 \%$ ). Par ailleurs des ventes sur Internet ont pu être identifiées.

Les autres noms commerciaux de spécialités (hors France) contenant du modafinil sont les suivants (liste non exhaustive) : Provigil $®$, Alertec $®$, Vigicer $($, Modalert ${ }^{\circledR}, \ldots$

\section{Posologie}

La posologie initiale recommandée est de 200 à $400 \mathrm{mg} / \mathrm{j}$. Le mode d'administration le plus fréquent est de répartir les doses entre le matin et le midi.

En cas d'insuffisance hépatique et/ou rénale, la posologie doit être diminuée de moitié.

\section{Méthodes de dépistage et dosage}

Peu de méthodes de dosage du modafinil ont été publiées dans la littérature depuis sa mise sur le marché.

La plupart des publications décrivent des méthodes de chromatographie en phase liquide couplée à une détection en ultraviolet (CLHP/UV).

Moachon (17) dose le modafinil et son métabolite acide sur $1 \mathrm{~mL}$ de plasma après extraction à $\mathrm{pH}$ acide par le diéthyl éther en présence d'un analogue fluoré du modafinil comme standard interne (laboratoire Lafon); la séparation chromatographique est réalisée sur une colonne $\mu$ Bondapak C8 $(10 \mu \mathrm{m}, 3,9 \times 300 \mathrm{~mm})$ de chez Waters, en mode isocratique ; la phase mobile est constituée d'un mélange acétonitrile : acide acétique glacial : eau distillée $(150: 12: 420, \mathrm{v} / \mathrm{v} / \mathrm{v})$; la détection se fait en UV à $236 \mathrm{~nm}$ (Limite de détection $(\mathrm{LD})=0,04 \mathrm{mg} / \mathrm{L}$, Limite de quantification $(\mathrm{LQ})=$ $0,13 \mathrm{mg} / \mathrm{L}$ ).

Burnat (18) propose le dosage du modafinil et de ses 2 métabolites inactifs (forme acide et sulfone) sur $1 \mathrm{~mL}$ de plasma acidifié après extraction sur cartouches $\mathrm{C} 18$ par le méthanol ; la séparation chromatographique en mode isocratique est effectuée dans un four thermostaté à $25^{\circ} \mathrm{C}$ en présence d'une précolonne $\mathrm{C} 8(5 \mu \mathrm{m}, 3,9 \mathrm{x}$ $30 \mathrm{~mm})$ et d'une colonne $\mathrm{C} 8(5 \mu \mathrm{m}, 3,9 \times 150 \mathrm{~mm})$ Symetry ; la phase mobile est constituée d'un mélange à $26 \%$ d'acétonitrile dans l'acide orthophosphorique $0,05 \mathrm{M}$ (ajusté à $\mathrm{pH} 2,6$ ) ; la détection se fait en UV à $225 \mathrm{~nm}(\mathrm{LD}=0,01 \mathrm{mg} / \mathrm{L}$ de modafinil ou $0,02 \mathrm{mg} / \mathrm{L}$ de métabolites, $\mathrm{LQ}=0,10$ et $0,12 \mathrm{mg} / \mathrm{l}$ respectivement).

Gorman (19) décrit une méthode CLHP/UV stéréospécifique qui permet la séparation des 2 énantiomères actifs du modafinil ; après extraction liquide/liquide de $1 \mathrm{~mL}$ de plasma par un mélange hexane : dichlorométhane : triéthyle amine $(55: 45: 2, \mathrm{v} / \mathrm{v} / \mathrm{v})$ en présence de 3,3-diphényl propylamine, le $\mathrm{D}$ et $\mathrm{L}$ modafinil sont séparés (en mode isocratique) sur une colonne chirale (EM séparations Chira Dex ${ }^{\mathrm{TM}} \beta$-cyclodextrin column) $(5 \mu \mathrm{m}, 4 \times 250 \mathrm{~mm})$ maintenue à $12^{\circ} \mathrm{C}$; la phase mobile est constituée d'un mélange tampon phosphate $0,02 \mathrm{M}, \mathrm{pH} 3$ / acétonitrile ( $84: 16, \mathrm{v} / \mathrm{v})$; la détection se fait en UV à $225 \mathrm{~nm}(\mathrm{LQ}=0,10 \mathrm{mg} / \mathrm{L}$ pour chaque énantiomère).

Gorman (20) modifie ensuite sa technique pour doser simultanément le modafinil et ses deux principaux métabolites à partir d'un volume de plasma réduit à $0,2 \mathrm{~mL}$. Après extraction liquide/liquide par un mélange hexane : dichlorométhane : acide acétique glacial (55: $45: 2, \mathrm{v} / \mathrm{v} / \mathrm{v})$ en présence d'acide phénylthioacétique comme standard interne ; la séparation est réalisée sur un colonne phényl Hypersilß BDS ,par un mélange isocratique de tampon phosphate (pH 2,5) méthanol $(64: 36$, v/v) et une détection UV à $235 \mathrm{~nm}$ (LQ $=0,1 \mathrm{mg} / \mathrm{L}$ ).

Schwertner (21) propose une autre méthode CLHP/UV appliquée au plasma et à l'urine avec l'acide phényl- 
thioacétique comme standard interne. Après extraction liquide/liquide de $2 \mathrm{~mL}$ de plasma par un mélange acétate d'éthyl : acide acétique $(100: 1, \mathrm{v} / \mathrm{v})$ ou de $5 \mathrm{~mL}$ d'urine par de l'acétate d'éthyl pur, la séparation chromatographique est réalisée sur colonne Symmetry C18 par une phase mobile composée d'un mélange méthanol/eau/acide acétique (500:500:1, v/v) (LQ = $0,1 \mathrm{mg} / \mathrm{L}$ à $233 \mathrm{~nm}$.).

McKinney (22) publie la première méthode de spectrométrie de masse, pour la détection du modafinil et de son métabolite acide dans les urines de chevaux; les 2 molécules peuvent être extraites à $\mathrm{pH}$ acide par un mélange de solvant hexane - dichlorométhane - acide acétique (55:45:2, v/v/v) ou bien par une extraction en phase solide (après acidification des urines à pH3) sur colonnes Abselute Nexus (60mg / $3 \mathrm{~mL}$ ) ; un lavage par la soude $0,1 \mathrm{M}$ et l'acide acétique $0,1 \mathrm{M}$ avant élution par l'acétate d'éthyle, permet d'obtenir des extraits plus propres ; le standard interne est le modafinil acide n-butyl ester. La séparation chromatographique est réalisée à température constante $\left(30^{\circ} \mathrm{C}\right)$ sur colonne Polaris Varian C18 ( $3 \mu \mathrm{m}, 2 \times 100 \mathrm{~mm})$ en présence d'une précolonne Phenomenex Sécurity Guard C18 $(2 \times 4 \mathrm{~mm})$. La phase mobile est constituée d'un mélange $\mathrm{A}$ : acide formique $25 \mathrm{mM} / \mathrm{B}$ : méthanol ; délivré selon un gradient linéaire TO : $5 \%$ de B, T9:95\% de $B$. La détection est réalisée en mode d'ionisation ESI. Des analyses de confirmation sont réalisées en móde full scan produit par ionisation dans la cellule de collision (CID) et la détection est basée sur la transition $167 \rightarrow 165(\mathrm{LD}=50 \mu \mathrm{g} / \mathrm{L})$. La limite de détection ainsi atteinte, permet de mettre en évidence le métabolite acide du modafinil dans les urines de chevaux, jusqu'à 4 jours après une administration.

La fragilité de cette molécule à haute température limite l'usage des méthodes de chromatographie en phase gazeuse au risque de travailler sur un fragment de la molécule. Cependant, tout récemment, Tseng (23) propose l'utilisation de la chromatographie gazeuse couplée à la spectrométrie de masse pour l'analyse d'échantillons d'urine humaine. Après extraction liquide liquide d' $1 \mathrm{~mL}$ d'urine par un mélange tertio-butylméthyl éther :2-propanol $(9: 1, \mathrm{v} / \mathrm{v})$ à un $\mathrm{pH} 9-9,5$ en présence de phénazine comme standard interne, la séparation chromatographique est réalisée sur une colonne HP5-MS® soumis au programme de température suivant : $90^{\circ} \mathrm{C}$ puis $15^{\circ} \mathrm{C} / \mathrm{mn}$ jusqu'à $240^{\circ} \mathrm{C}$. La quantification est basée sur le fragment $\mathrm{m} / \mathrm{z} 167(\mathrm{LD}=$ $0.109 \mathrm{mg} / \mathrm{L}$ et $\mathrm{LQ}=0,363 \mathrm{mg} / \mathrm{L}$ ). L'auteur n'évoque pas de problèmes particuliers concernant la possible thermo-dégradation du modafinil. Par ailleurs, il n'explique pas le choix du $\mathrm{pH}$ d'extraction basique.

\section{Cas cliniques}

Les cas cliniques d'intoxication au modafinil sont rarissimes.

Des cas d'effets indésirables comme l'exacerbation d'un état psychotique (24), des troubles du rythme (25) sont signalés.

Une publication récente (26) rapporte le cas de 2 patients pour lesquels le modafinil aurait induit irritabilité et agression.

Un cas de prescription abusive révélé à la survenue du décès d'une patiente est en cours de publication (27). La prescription associait modafinil, méthylphénidate, clobazam, lormétazépam et mépronizine.

Par ailleurs, des cas de dopage au modafinil ont été rapportés dans le milieu de l'athlétisme en 2003 (28), en particulier dans l'équipe américaine : la sprinteuse Kelli White qui a remporté les médailles d'Or du 100 et 200 mètres aux Championnats du Monde à Paris en Août 2003, et le sprinteur Calvin Harrisson, relayeur du $4 \times 400 \mathrm{~m}$ aux mêmes championnats. Et toujours dans l'athlétisme, trois autres sportifs américains, Chryste Gaines, Sandra Glover et Eric Thomas, auraient également été contrôlés positifs au modafinil lors du championnat US en juin 2003 ou lors des sélections américaines de Palo Alto la même année. Le modafinil est inscrit depuis 2004 sur la liste des substances et méthodes interdites en compétition du code mondial anti-dopage (29).

Enfin, tout récemment en décembre 2005, il est dévoilé dans la presse nationale (30) que l'armée française aurait « administré », du modafinil lors de la guerre contre l'Irak en 1991, sans suivre la procédure légale d'un essai clinique. Son rôle éventuel, parmi d'autres causes, dans la survenue d'un «syndrome de la guerre du Golfe » fait l'objet d'une instruction judiciaire.

\section{Autres indications en cours d'expérimentation}

Depuis janvier 2004, deux nouvelles indications du modafinil ont été approuvées aux USA, il s'agit du traitement de la somnolence excessive associée au SAHOS et des troubles du sommeil liés au travail posté (31-33). Par ailleurs, de nombreuses études expérimentales et des essais cliniques sont en cours pour évaluer de nouvelles indications telles que :

- le trouble déficitaire de l'attention chez l'enfant (34), - la fatigue associée à des pathologies telles que la sclérose en plaque (5) la sclérose latérale amyotrophique (35) ou la dépression (36), 
- l'aide au sevrage à la cocaïne $(37,38)$.

Enfin, les militaires s'intéressent de près aux effets du modafinil dans l'amélioration des performances en cas de manque de sommeil (39-42) mais également sur son éventuel effet néfaste face à d'autres types de « stress » comme la chaleur excessive (43).

\section{Dérivés du modafinil ou autres formes en cours d'expérimentation}

L'adrafinil est commercialisé en France sous le nom d'Olmifonß et proposé dans le traitement des troubles de la vigilance et de l'attention ainsi que du ralentissement idéomoteur des sujets âgés (2). Il se métabolise en modafinil mais il n'a pas, en l'état actuel du dossier fait la preuve de son efficacité en dehors du sujet âgé. L'Olmifon (B) est actuellement soumis aux règles de prescription de la liste $\mathrm{I}$.

Le laboratoire Cephalon qui commercialise actuellement le modafinil (précédemment distribué par le laboratoire Lafon), a fait enregistrer récemment un nouveau dérivé auprès de la FDA (Food and Drug Administration) qui consiste en l'isomère soit le $\mathrm{r}$ modafinil (ou armodafinil) sous le nom commercial de Nuvigilß.

\section{Conclusion}

Actuellement, les cas d'intoxications ou d'abus au modafinil sont peu nombreux dans la littérature. Les règles de prescription très strictes avec lesquelles ce produit est distribué sont certainement à prendre en compte dans ce constat mais ceci ne doit pas conduire à sous-estimer ses risques de pharmacodépendance et de mésusage. Rappelons que son dépistage analytique nécessite une extraction en milieu acide suivie d'une analyse par chromatographie liquide ce qui n'est pas forcément une procédure appliquée systématiquement dans les laboratoires de toxicologie. On peut poser l'hypothèse d'un certain nombre de cas cliniques encore non détectés. Enfin si l'usage du modafinil doit également être élargi à d'autres indications comme cela est observé dans d'autres pays, la vigilance face à un risque de conduite addictive, devra rester constante.

\section{Références}

1. Giles J. Alertness drug arouses fears about "lifestyle" misuse. Nature $2005 ; 436: 1076$.

2. Vidal 2005. http://www.vidalpro.net/

3. Puech A., Golbenberg F. Modafinil : Modiodal(r). Le traitement de la narcolepsie et de l'hypersomnie idiopathique. Comité d'Education Sanitaire et Sociale de la Pharmacie française. Fiche technique, mai 1995.

4. Dauvilliers Y., Carlander B., Billiard M. La narcolepsie, de Westphal à l'hypocrétine. Presse Med. 2004 ; 33 : 1593-600.

5. Billiard M. Le modafinil : pharmacologie et perspectives thérapeutiques. Rev. Neurol. $2003 ; 159: 122-5$.

6. Moffat A.C., Osselton M.D., Widdop B. Clarke's analysis of drugs and poisons. Third edition, $2004 ; 1294-5$.

7. O'Neil M.J., Smith A., Heckelman P.E., Modafinil in The Merck Index. Thirteenth edition 2001; 1112.

8. Baselt R.C. Disposition of toxic drugs and chemicals in man. Foster City : biomedical publications 2004 : 756-7.

9. Randall DC, Shneerson IM, File SE. Cognitive effects of modafinil in student volunteers may depend on IQ. Pharmacol. Biochem. Behav. 2005 ; 82(1) : 133-9.

10. Wesensten NJ, Killgore WD, Balkin TJ. Performance and alertness effects of caffeine, dextroamphetamine, and modafinil during sleep deprivation. J. Sleep Res. 2005 ; $14(3): 255-66$.

11. Fiche d'information thérapeutique modafinil http:/www.admi.net/jo/19991107/MESS9923344A.html

12. Jasinski DR, Kovacevic-Ristanovic R. Evaluation of the abuse liability of modafinil and other drugs for excessive daytime sleepiness associated with narcolepsy. Clin. Neuropharmacol. 2000 ; 23(3) : 149-56.

13. Laboratoire Lafon. Modiodal@ modafinil : dossier d'information médicale et pharmaceutique. 1994.

14. Lagarde M., Milhaud C. Electroencephalographic effects of modafinil, an alpha-1-adrenergic psychostimulant, on the sleep of Rhesus Monkeys. Sleep. 1990 ; 13(5) : 441-8.

15. Jasinski DR. An evaluation of the abuse potential of modafinil using methylphenidate as a reference. J. Psychopharmacol. $2000 ; 14(1)$ : 53-60.

16. Comité de coordination des vigilances des produits de santé. Bilan 2003 : principaux faits marquants. http://agmed.sante.gouv.fr/pdf/5/bilan04.pdf

17. Moachon G, Matinier D. Simultaneous determination of modafinil and its acid metabolite by high-performance liquid chromatography in human plasma. J. Chromatogr. B Biomed. Appl. 1994 ; 654(1) : 91-6.

18. Burnat P, Robles F, Do B. High-performance liquid chromatographic determination of modafinil and its two metabolites in human plasma using solid-phase extraction. J. Chromatogr. B Biomed. Sci. Appl. 1998 ; 706(2) : 295 304.

19. Gorman SH. Determination of the D-and L-enantiomers of modafinil in human plasma utilizing liquid-liquid extraction and high-performance liquid chromatography. J. Chromatogr. B Biomed. Sci. Appl. 1999 ; 730(1) : 1-7. 
20. Gorman SH. Determination of modafinil, modafinil acid and modafinil sulfone in human plasma utilizing liquidliquid extraction and high-performance liquid chromatography. J. Chromatogr. B Analyt. Technol Biomed Life Sci. $2002 ; 767(2): 269-76$.

21. Schwertner HA, Kong SB. Determination of modafinil in plasma and urine by reversed phase high-performance liquid-chromatography. J. Pharm. Biomed. Anal. 2005 ; 37(3) : 475-9.

22. McKinney AR, Suann CJ, Stenhouse AM. The detection of modafinil and its major metabolite in equine urine by liquid chromatography/mass spectrometry. Rapid Commun Mass Spectrom. 2005 : 19(10) : 1217-20.

23. Tseng YL, Uralets V. Lin CT, Kuo FH. Detection of modafinil in human urine by gas chromatography-mass spectrometry. J. Pharm Biomed. Anal. 2005 ; 39(5) : 1042-5.

24. Mariani JJ, Hart CL. Psychosis associated with modafinil and shift work. Am J Psychiatry. $2005 ; 162(10)$ : 1983.

25 . Oskooilar N. A case of premature ventricular contractions with modafinil. Am J Psychiatry. 2005 ; 162(10) : 1983-4.

26. Ranjan S, Chandra PS. Modafinil-induced irritability and aggression? A report of 2 bipolar patients. J. Clin. Psychopharmacol. $2005 ; 25(6): 628-9$.

27. Pelissier-Alicot A.L., Piercecchi-Marti M.D., Bartoli C., Kuhlmann E., Coiffait P.E., Sanvoisin A., Giocanti D., Leonetti G. Abusive prescription of psychostimulants : a study of two cases. J. Forensic Sci. Sous presse.

28. Kaufman KR. Modafinil in sports: ethical considerations. Br. J. Sports Med. 2005 ; 39(4) : 241-4.

29. Liste des méthodes et substances interdites en compétition pour l'année 2006. Code Mondial Antidopage. http://www.wada-ama.org/rtecontent/document/2006 _LIST_French.pdf

30. Bordenave Y., Prieur C. Les cobayes de la guerre du Golfe. Le Monde $2005 ; 18942$ : 3 .

31. Schwartz JR. Modafinil: new indications for wake promotion. Expert. Opin. Pharmacother. $2005 ; 6(1)$ : 115-29.

32. Keating GM, Raffin MJ. Modafinil : a review of its use in excessive sleepiness associated with obstructive sleep apnoea/hypopnoea syndrome and shift work sleep disorder.CNS Drugs. 2005 ; 19(9) : 785-803.

33. Czeisler CA, Walsh JK, Roth T, Hughes RJ, Wright KP, Kingsbury L, Arora S, Schwartz JR, Niebler GE, Dinges DF; U.S. Modafinil in Shift Work Sleep Disorder Study Group. Modafinil for excessive sleepiness associated with shift-work sleep disorder. N Engl J Med. 2005 Aug 4 ; 353(5) : 476-86.
34. Biederman J, Swanson JM, Wigal SB, Kratochvil CJ, Boellner SW, Earl CQ, Jiang J, Greenhill L. Efficacy and safety of modafinil film-coated tablets in children and adolescents with attention-deficit/hyperactivity disorder: results of a randomized, double-blind, placebo-controlled, flexible-dose study. Pediatrics. $2005 ; 116(6)$ : 777-84.

35. Carter GT, Weiss MD, Lou JS, Jensen MP, Abresch RT, Martin TK, Hecht TW, Han JJ, Weydt P, Kraft GH. Modafinil to treat fatigue in amyotrophic lateral sclerosis : an open label pilot study. Am J Hosp Palliat Care. 2005 ; 22(1) : 55-9.

36. Price CS, Taylor FB. A retrospective chart review of the effects of modafinil on depression as monotherapy and as adjunctive therapy. Depress. Anxiety. 2005 ; 21(4) : 14953.

37. Donovan JL, DeVane CL, Malcolm RJ, Mojsiak J, Chiang CN, Elkashef A, Taylor RM. Modafinil influences the pharmacokinetics of intravenous cocaine in healthy cocaine-dependent volunteers. Clin Pharmacokinet. 2005 ; 44(7) : 753-65.

38. Dackis CA, Kampman KM, Lynch KG, Pettinati HM, O'Brien CP. A double-blind, placebo-controlled trial of modafinil for cocaine dependence. Neuropsycho- pharmacology. $2005 ; 30(1): 205-11$.

39. Stivalet P., Esquivié D., Barraud P.A., Leifflen D., Raphel C. Effecs of modafinil on attentional processes during 60 hours of sleep deprivation. Hum. Psychopharmacol. Clin. Exp. $1998 ; 13: 501-7$.

40. Chapotot F, Pigeau R, Canini F, Bourdon L, Buguet A. Distinctive effects of modafinil and d-amphetamine on the homeostatic and circadian modulation of the human waking EEG Psychopharmacology 2003 ; 166(2) : 127-38.

41. Westcott KJ. Modafinil, sleep deprivation, and cognitive function in military and medical settings. Mil. Med. $2005 ; 170(4): 333-5$.

42. Caldwell JA, Caldwell JL. Fatigue in military aviation: an overview of US military-approved pharmacological countermeasures. Aviat. Space Environ. Med. 2005 ; 76 (7 Suppl) : C39-51.

43. Launay JC, Besnard Y, Guinet A, Bessard G, Raphel C, Savourey G. Effects of modafinil on heat thermoregulatory responses in humans at rest. Can J Physiol Pharmacol. $2002 ; 80(8)$ : 796-803. 